\section{Cor/raça no Estudo Pró-Saúde: resultados comparativos de dois métodos de autoclassificação no Rio de Janeiro, Brasil}

\author{
Ethnicity/race in the Pró-Saúde Study: \\ comparative results of two methods \\ of self-classification in Rio de Janeiro, Brazil
}

\author{
Marcos Chor Maio 1 \\ Simone Monteiro 2 \\ Dóra Chor 3 \\ Eduardo Faerstein 4 \\ Claudia S. Lopes 4
}

\section{Introdução}

1 Casa de Oswaldo Cruz, Fundação Oswaldo Cruz, Rio de Janeiro, Brasil. 2 Instituto Oswaldo Cruz, Fundação Oswaldo Cruz, Rio de Janeiro, Brasil.

3 Escola Nacional de Saúde Pública, Fundação Oswaldo Cruz, Rio de Janeiro, Brasil. 4 Instituto de Medicina Social, Universidade do Estado do Rio de Janeiro, Rio de Janeiro, Brasil.

Correspondência M. C. Maio Departamento de Pesquisa, Casa de Oswaldo Cruz, Fundação Oswaldo Cruz. Av. Brasil 4036, Rio de Janeiro, $R J$ 21040-361, Brasil. maio@altemex.com.br
Variations in classification systems related to color/race and the contextual dependence of their utilization are two challenges for researchers conducting health studies within an ethnic/racial framework. A comparison was made between the results obtained by two distinct approaches to racial self-classification - $a$ closed question (using categories from the IBGE, or National Census Bureau) and an open-ended question - in a cohort study of employees at a university in Rio de Janeiro. According to the closed question, $54.0 \%$ of the 3,717 respondents classified themselves as white, $30.0 \%$ as brown (or mixed-race), and 16.0\% as black. According to answers to the open-ended question, the proportions were $53.0 \%, 25.0 \%$, and $22.0 \%$, respectively, when the terms "moreno", "mestiço", and "mulato" were grouped under the "brown" category. Despite the high level of agreement (kappa = 0.80; 95\%CI: 0.78-0.82), a sizeable numbers of black and brown respondents (open-ended question) chose "whitening" categories available in the list supplied by the IBGE. The use of ethnic/racial categories in health studies can both reveal information on health inequalities in Brazil and also contribute to the formulation of public health policies adequately informed by the specificities of Brazilian society.

Ethnics Groups; Cohort Studies; Health Inequity
As relações entre raça/etnia e os agravos à saúde têm sido abordadas pela produção acadêmica internacional, particularmente a norteamericana, mas ainda ocupa reduzido espaço na literatura brasileira 1 . Análises recentes identificam que a escassez de pesquisas desenvolvidas na área da saúde pública no Brasil com recorte racial/étnico deve-se, em parte, à subordinação dessa dimensão a determinantes sócio-econômicos. Não menos importante é a opinião, ainda comumente aceita, de que o Brasil sofreria de reduzidos graus de preconceito e discriminação raciais, gerando, com isso, a crença de que o racismo tem pouca relevância para o entendimento não apenas da sociabilidade à brasileira, mas também das iniqüidades sociais existentes no país 2,3. Constata-se, entretanto, que nos últimos anos é crescente o interesse pelo estudo das relações entre raça/etnia e saúde, com ênfase na população negra, entre pesquisadores do campo da saúde coletiva e militantes de movimentos sociais.

Vale assinalar que a preocupação em se analisar as implicações das disparidades raciais sobre a ocorrência dos agravos à saúde, surge no momento em que se aprofunda a discussão sobre o racismo no Brasil e sobre os supostos limites das políticas públicas universalistas, que não teriam levado em consideração as especificidades de determinados grupos so- 
ciais. Tal debate guarda relação com a ascensão do movimento social negro e com a importância que o combate ao racismo vem assumindo na agenda de instituições intergovernamentais (Organização das Nações Unidas - ONU/Organização das Nações Unidas para a Educação, a Ciência e a Cultura - UNESCO/Organização Pan-Americana da Saúde - OPAS), de fundações filantrópicas norte-americanas e agências nacionais de fomento à pesquisa, responsáveis pelo apoio a diversas investigações e/ou iniciativas sociais e políticas direcionadas para a redução das desigualdades raciais na sociedade brasileira. Paralelamente, militantes do movimento negro conquistaram nichos de atuação no aparato estatal, particularmente nas gestões de Fernando Henrique Cardoso (1994-2002) e do atual presidente Luiz Inácio Lula da Silva, na qual foi criada a Secretaria de Promoção da Igualdade Racial 4.

É nesta conjuntura social e política que adquire maior destaque o debate sobre a criação de um campo de pesquisa e de intervenção voltado para a saúde da população negra, que inclui a produção de conhecimento científico, a capacitação de profissionais de saúde, a divulgação de informações para a população e a atenção à saúde. As discussões que fundamentam esta proposição têm por base análises sobre as repercussões do racismo na produção das desigualdades em saúde e a relevância da caracterização de doenças étnico/raciais, seja em relação à sua determinação genética, seja em termos da sua dimensão sócio-econômica 5 .

Pondera-se que as investigações sobre as associações entre raça e saúde devem estar calcadas nos estudos sociológicos, que evidenciam que as possibilidades de mobilidade social vertical para pretos e pardos permanecem muito aquém das chances dos brancos, mesmo quando se controlam as variáveis ligadas à origem social. Tais estudos observam que o caráter assimétrico das relações raciais é um fator fundamental na determinação das desigualdades sociais no Brasil 6,7,8. Dentro desta perspectiva, cabe desenvolver pesquisas sobre as implicações da discriminação étnico/racial sobre a ocorrência de agravos à saúde 9 .

A hipótese da influência de fatores genéticos na determinação de doenças relacionadas a grupos étnicos/raciais tem suscitado indagações de ordem metodológica e conceitual. Um dos aspectos controversos diz respeito à irre dutibilidade dos agravos à saúde à sua dimensão biológica, visto que as doenças, em geral, também decorrem de fatores históricos, econômicos e psicossociais, cuja inclusão em modelos abrangentes de causalidade é ainda insu- ficiente. Argumenta-se ainda que a produção científica na área da saúde não compartilha de uma definição comum acerca das categorias raça e etnia, e que as implicações dessa falta de consistência conceitual devem ser consideradas na interpretação das diferenças nos indicadores de saúde entre os grupos étnico/raciais 1 . Entretanto, as críticas relativas à vinculação entre determinantes genéticos e grupos étnico/raciais e à imprecisão dos conceitos de raça e etnia, não devem desestimular a reflexão sobre as implicações do fenômeno do racismo, enquanto princípio classificatório e fator estruturante da estratificação social, para a compreensão das iniqüidades na área da saúde.

Um dos tópicos realçados na literatura sobre o binômio raça/etnia e saúde diz respeito às variações nos sistemas de classificação dos referidos grupos nas estatísticas populacionais, tanto no caso norte-americano 10 , cuja classificação se orienta pelo conceito de ancestralidade e pelo modelo bipolar (branco e negro), quanto na experiência brasileira, onde a diversidade do sistema de classificação decorre da combinação de aspectos fenotípicos com atributos de classe, status e educação. De fato, o padrão classificatório no Brasil é freqüentemente caracterizado pela fluidez (indeterminação, subjetividade e dependência contextual) em sua aplicação. Constata-se, por exemplo, que quanto maior a posição sócio-econômica do indivíduo, mais recorrente é a possibilidade dele se "embranquecer" 11. Deve ser salientado que, não obstante a existência de um amplo leque de denominações da cor/raça na sociedade brasileira, pesquisas que comparam a autoclassificação da pergunta aberta com a autoclassificação segundo as categorias oficiais do Censo Demográfico, revelam que cerca de $95,0 \%$ da população utilizam apenas seis termos na pergunta aberta, e ainda que há grande concordância entre as respostas aos dois tipos de abordagem 12,13 .

Admitindo-se que os erros de medida são uma preocupação constante na classificação de grupos segundo qualquer característica, pretende-se salientar a importância do exame das possíveis estratégias para inclusão do recorte étnico/racial na descrição do perfil de morbimortalidade da população brasileira, a exemplo do que já foi realizado no âmbito da educação, renda e mercado de trabalho ${ }^{8}$. Diferentes métodos de classificação racial têm sido avaliados, em estudos de saúde, no Brasil e em outros países 14,15,16. Alguns autores consideram que a autoclassificação (comparada à categorização realizada por entrevistadores) é o método que melhor reflete a identificação do indiví- 
duo com os diversos grupos raciais 16,17. Outros apontam que a melhor abordagem depende do objetivo do estudo. Assim, por exemplo, no caso de investigações sobre a discriminação racial, observadores externos expressariam melhor a forma como o indivíduo é "visto" pela sociedade (Williams, 2002, apud Kaplan \& Bennett 18).

É neste contexto que o presente trabalho se insere. Pretende-se analisar a autoclassificação de raça baseando-se em duas abordagens distintas, e suas implicações para os estudos na área da saúde. As análises foram conduzidas com base em dados do Estudo Pró-Saúde, que tem como objetivo investigar o papel de ampla gama de determinantes sociais e fontes de desigualdades (e.g. condições sócio-econômicas precoces e tardias, gênero, cor/raça), mobili dade social, eventos de vida produtores de estresse e padrões de apoio social, sobre a morbidade física e mental de uma coorte de funcionários de uma universidade no Rio de Janeiro. Tem-se assim o propósito de analisar em que medida o sistema de classificação racial pode influenciar a investigação de associações entre condições de saúde e os respectivos grupos étnico/raciais em populações brasileiras.

\section{Métodos}

\section{Participantes}

O Estudo Pró-Saúde é uma pesquisa de coorte dos funcionários técnico-administrativos do quadro efetivo de uma universidade no Rio de Janeiro. Na primeira etapa de coleta de dados (agosto/outubro de 1999), todos os 4.448 funcionários técnico-administrativos foram convidados a participar. Destes, 4.030 (90,6\% da população alvo) participaram do estudo.

Nas análises apresentadas neste artigo, foram excluídos quatro funcionários com 80 anos de idade ou mais, já que o grupo etário com contingente suficiente de participantes encontrase entre 20 e 70 anos de idade. Dos 4.026 participantes, foram também excluídos aqueles que deixaram de responder a uma das duas perguntas para autoclassificação de raça fechada (56/ $4.026=1,4 \%)$ e aberta $(136 / 4.026=3,4 \%)$; aqueles cujas respostas à pergunta aberta, não foi passível de classificação $(36 / 4.026=0,9 \%)$; e os participantes que se autoclassificaram como indígenas ou amarelos na pergunta fechada (101/ $4.026=2,5 \%)$ e/ou na pergunta aberta $(18 / 4.026=$ $0,5 \%)$. Dessa forma, foram comparadas as respostas, aos dois tipos de autoclassificação de raça, de 3.717 participantes (2.086 mulheres) que representam $92,0 \%$ da população inicial.

\section{Medidas de interesse}

Os participantes responderam, nos locais de trabalho, a questionário multidimensional $\mathrm{e}$ autopreenchível. O questionário continha dois itens distintos para a autoclassificação de raça. Em um dos blocos iniciais de perguntas foi incluída questão aberta onde qualquer termo desejado pelo funcionário poderia ser registrado (“Em sua opinião, qual é sua cor ou raça?”). Ao final do questionário, simulou-se a situação proposta pelo Censo Demográfico do Instituto Brasileiro de Geografia e Estatística - IBGE (“O Censo Brasileiro usa os termos preta, parda, branca, amarela e indígena para classificar a cor ou raça das pessoas. Se você tivesse que responder ao Censo do IBGE hoje, como se classificaria a respeito de sua cor ou raça?"), com suas opções de resposta (preta, parda, branca, amarela, indígena).

Cabe esclarecer que a ordenação das perguntas aberta e fechada, inseridas num amplo questionário, teve por objetivo evitar a indução das categorias censitárias nas respostas.

Outras características sócio-demográficas foram incluídas na análise: idade, sexo, escolaridade, renda líquida familiar e número de pessoas dependentes dessa renda. A renda familiar per capita foi calculada por meio da divisão da renda líquida familiar no mês anterior à pesquisa pelo número de pessoas que dependiam dessa renda para viver. Esse resultado foi transformado em número de salários mínimos (SM), cujo valor era de $\mathrm{R} \$ 136,00$ à época da pesquisa.

\section{Análise de dados}

Os participantes utilizaram quarenta e um termos distintos para identificar sua cor/raça. Esses termos foram agrupados inicialmente em sete categorias, correspondendo a 99,0\% dos participantes: branco (e.g. branco, clara, pele clara); pardo (e.g. pardo, pardo claro); moreno (e.g. moreno, moreno claro, moreno escuro, moreno jambo); mulato (e.g. mulato, mulato claro, mulato escuro); mestiço (e.g. mestiço, miscigenado, caboclo, misto, mameluco, híbrido), negro, preto (e.g. preto, pele escura, africano). A etapa seguinte consistiu no agrupamento daquelas sete categorias em três: branco, pardo (pardo, moreno, mulato, mestiço, caboclo) e preto (negro, preto, africano, escuro).

Para avaliação da consistência entre as respostas aos dois itens, foram estimadas proporções de concordância e estatísticas kappa 19 entre as categorias branco, pardo e preto obtidas diretamente da pergunta fechada e as mesmas categorias obtidas pelo agrupamento das 
respostas à pergunta aberta, para homens e mulheres separadamente. Comparações segundo estratos de idade, escolaridade e renda familiar per capita complementaram a análise.

\section{Resultados}

A idade dos participantes variou entre 22 e 70 anos, concentrando-se entre 30 e 49 anos de idade (cerca de 70,0\%), e pouco mais da metade $(55,0 \%)$ eram do sexo feminino. As mulheres apresentaram maior nível de escolaridade: $46,0 \%$ relataram haver completado o terceiro grau e $34,0 \%$ o segundo, comparadas a $33,0 \%$ e $39,0 \%$ dos homens, respectivamente. Funcionários lotados no hospital universitário constituíam cerca da metade $(48,0 \%)$ dos participantes, o restante se concentrava no campus central, localizado na capital do Estado do Rio de Janeiro, com pequena proporção nos campi regionais.

Comparando-se as sete categorias resultantes do agrupamento das respostas à pergunta aberta com as categorias da pergunta fechada, entre os homens, a maior proporção de concordância foi observada entre os 878 partici pantes que se identificaram como brancos e os 239 que se identificaram como pardos (pergunta aberta): $96,0 \%$ e 95,0\%, respectivamente, escolheram estas mesmas categorias entre as opções da pergunta fechada (Tabela 1). Entre aqueles que se identificaram como pretos ou negros (pergunta aberta), 76,0\% e 69,0\%, respectivamente, optaram pela categoria de cor/raça preta na pergunta fechada; ou seja, cerca de $24,0 \%$ daqueles que se identificaram como pretos e
$31,0 \%$ entre aqueles autoclassificados como negros escolheram a cor/raça parda entre as categorias fechadas.

Em relação aos participantes que se identificaram como morenos ou mestiços (pergunta aberta), 75,0\% e 71,0\%, respectivamente, classificaram-se como pardos. Entre os mulatos, 85,0\% incluíram-se nesta categoria. Cabe salientar que nestes subgrupos intermediários, 23,0\% dos morenos e $25,0 \%$ dos mestiços classificaram-se como brancos, e apenas 5,0\% dos mulatos optaram por essa categoria na pergunta fechada.

Comportamento bastante semelhante foi observado entre as mulheres (Tabela 1). As maiores proporções de concordância foram estimadas entre as participantes que se identificaram, na pergunta aberta, como pardas $(97,0 \%)$ ou brancas $(96,0 \%)$. Entre aquelas que se identificaram como pretas ou negras (pergunta aberta), cerca de um quarto optou por parda entre as categorias fechadas.

A tendência observada entre os homens morenos ou mestiços foi ainda mais marcante entre as mulheres: nenhuma participante que se identificou como mulata optou pela categoria branca da pergunta fechada, mas cerca de $28,0 \%$ das morenas e 33,0\% das mestiças escolheram esta alternativa.

Nova comparação foi realizada após agrupamento das respostas relativas aos termos pardo, moreno, mulato e mestiço na categoria parda, e os termos preto e negro na categoria preto. De acordo com a pergunta fechada, 54,0\% dos 3.717 participantes classificaram-se como brancos, $30,0 \%$ como pardos e $16,0 \%$ como pretos. De acordo com a pergunta aberta, essas

Tabela 1

Comparação entre autoclassificação de cor/raça segundo pergunta aberta e pergunta fechada, por sexo. Estudo Pró-Saúde, Rio de Janeiro, Brasil, 1999.

\begin{tabular}{|c|c|c|c|c|c|c|c|c|}
\hline \multirow{2}{*}{$\begin{array}{l}\text { Pergunta fechada } \\
\text { Homens }\end{array}$} & \multicolumn{8}{|c|}{ Pergunta aberta $(\%)^{\star}$} \\
\hline & Brancos & Pardos & Morenos & Mulatos & Mestiços & Pretos & Negros & Total \\
\hline Brancos & 96,0 & 1,3 & 23,0 & 5,1 & 25,0 & 0,0 & 0,0 & 895 \\
\hline Pardos & 3,9 & 95,4 & 74,7 & 84,6 & 71,4 & 23,9 & 31,3 & 527 \\
\hline Pretos & 0,1 & 3,3 & 2,3 & 10,3 & 3,6 & 76,1 & 68,7 & 209 \\
\hline Total $(100,0 \%)$ & 878 & 239 & 174 & 39 & 28 & 46 & 227 & 1.631 \\
\hline Mulheres & Brancas & Pardas & Morenas & Mulatas & Mestiças & Pretas & Negras & Total \\
\hline Brancas & 95,9 & 0,4 & 27,7 & 0,0 & 33,3 & 1,8 & 0,0 & 1.113 \\
\hline Pardas & 4,1 & 96,9 & 70,9 & 94,6 & 63,0 & 21,8 & 27,8 & 577 \\
\hline Pretas & 0,0 & 2,7 & 1,4 & 5,4 & 3,7 & 76,4 & 72,2 & 396 \\
\hline Total $(100,0 \%)$ & 1.099 & 226 & 141 & 37 & 54 & 55 & 474 & 2.086 \\
\hline
\end{tabular}

* Proporção em relação aos totais relativos à pergunta aberta. 
proporções foram $53,0 \%, 25,0 \%$, e $22,0 \%$, respectivamente (Tabela 2 ).

Apesar da concordância elevada estimada pelo coeficiente kappa entre as respostas dos homens (kappa =0,81; IC95\%: 0,78-0,83) e das mulheres (kappa =0,80; IC95\%: 0,77-0,82), proporções não desprezíveis dos participantes que se identificaram como negros ou pardos na pergunta aberta, escolheram categorias mais "claras” na autoclassificação de raça proposta pela pergunta fechada (Tabela 3). Assim, 30,0\% e $27,0 \%$ dos homens e mulheres, respectivamente, que se identificaram como negros, optaram pela categoria parda na pergunta fechada. Entre os pardos (pergunta aberta), cerca de $11,0 \%$ dos homens e $13,0 \%$ das mulheres classificaram-se como brancos. Tal tendência foi também observada na pergunta fechada, nos estratos da população definidos segundo idade, escolaridade e renda familiar per capita (Tabela 4). Por exemplo, entre os homens com menos de 40 anos de idade, $34,0 \%$ daqueles que se identificaram como negros classificaram-se como pardos na pergunta fechada. Dentre aqueles que se identificaram como pardos, $10,0 \%$ classificaram-se como brancos. Comportamento bastante semelhante foi identificado entre os participantes com 40 anos ou mais e em ambos os grupos etários femininos.

Comparando-se os estratos de escolaridade, algumas diferenças importantes foram encontradas. Entre as mulheres (mas não entre os homens), a proporção de negras e pardas (pergunta aberta) que se classificaram na pergunta fechada como pardas e brancas, respectivamente, aumentou com o grau de escolaridade. Considerando-se apenas os participantes com o primeiro grau, cerca de um terço dos homens que se identificaram como negros classificaram-se como pardos na pergunta fechada, e dentre os pardos, $10,0 \%$ classificaram-se como brancos. Estas proporções foram bem menores entre as mulheres com o mesmo grau de instrução: $18,0 \%$ das negras e 3,0\% das pardas optaram por parda ou branca, respectivamente, na pergunta aberta.

A proporção de participantes que se identificaram como negros na pergunta aberta e que se classificaram como pardos na pergunta fechada não apresentou variação importante nos diferentes estratos de renda familiar per capita, situando-se em tomo de 30,0\%. Entre aqueles que se identificaram como pardos, no entanto, a proporção de participantes que se classificaram como brancos, na pergunta fechada, aumentou conforme o nível de renda, especialmente entre as mulheres: $7,0 \%(<3 \mathrm{SM}), 12,0 \%$ (3-6 SM) e 21,0\% (> $6 \mathrm{SM}$ ).
Tabela 2

Proporção de brancos, pardos e pretos segundo perguntas fechada e aberta, por sexo.

Estudo Pró-Saúde, Rio de Janeiro, Brasil, 1999.

\begin{tabular}{lcc}
\hline & $\begin{array}{c}\text { Pergunta } \\
\text { fechada (\%) }\end{array}$ & $\begin{array}{c}\text { Pergunta } \\
\text { aberta (\%) }\end{array}$ \\
\hline Homens & 54,9 & 53,8 \\
Brancos & 32,3 & 29,4 \\
Pardos & 12,8 & 16,7 \\
Pretos & & \\
Mulheres & 53,4 & 52,7 \\
Brancas & 27,7 & 22,0 \\
Pardas & 19,0 & 25,4 \\
Pretas & & \\
Total & 54,0 & 53,2 \\
Brancos & 29,7 & 25,2 \\
Pardos & 16,3 & 21,6 \\
Pretos & &
\end{tabular}

Tabela 3

Proporção de concordância (\%) entre autoclassificação de cor/raça de acordo com pergunta aberta e pergunta fechada, por sexo. Estudo Pró-Saúde, Rio de Janeiro, Brasil, 1999.

\begin{tabular}{|c|c|c|c|c|}
\hline \multirow[t]{2}{*}{ Pergunta fechada } & \multicolumn{3}{|c|}{ Pergunta aberta (\%) } & \multirow[t]{2}{*}{ Карра (IC95\%) } \\
\hline & Brancos & Pardos* & Pretos ${ }^{\star \star}$ & \\
\hline Homens & & & & $0,81(0,78-0,83)$ \\
\hline Brancos & 96,0 & 10,8 & 0,0 & \\
\hline Pardos & 3,9 & 85,6 & 30,0 & \\
\hline Pretos & 0,1 & 3,5 & 70,0 & \\
\hline Total (n) & 878 & 480 & 273 & \\
\hline Mulheres & & & & $0,80(0,77-0,82)$ \\
\hline Brancas & 95,9 & 12,7 & 0,2 & \\
\hline Pardas & 4,1 & 84,7 & 27,2 & \\
\hline Pretas & 0,0 & 2,6 & 72,6 & \\
\hline Total (n) & 1.099 & 458 & 529 & \\
\hline Toda a população & & & & $0,80(0,78-0,82)$ \\
\hline Brancos & 96,0 & 11,7 & 0,1 & \\
\hline Pardos & 4,0 & 85,2 & 28,2 & \\
\hline Pretos & 0,1 & 3,1 & 71,7 & \\
\hline Total (n) & 1.977 & 938 & 802 & \\
\hline
\end{tabular}

* Agrupamento das categorias: pardos, morenos, mulatos e mestiços.

** Agrupamento das categorias: negros e pretos. 
Proporção de concordância (\%) entre autoclassificação de cor/raça de acordo com pergunta aberta e pergunta fechada, segundo escolaridade, renda per capita e idade. Estudo Pró-Saúde, Rio de Janeiro, Brasil, 1999.

\begin{tabular}{|c|c|c|c|c|c|c|c|c|c|}
\hline \multirow{3}{*}{$\begin{array}{l}\text { Pergunta } \\
\text { fechada }\end{array}$} & \multicolumn{9}{|c|}{ Pergunta aberta } \\
\hline & \multicolumn{3}{|c|}{ Homens } & \multicolumn{3}{|c|}{ Mulheres } & \multicolumn{3}{|c|}{ Total } \\
\hline & Brancos & Pardos & Pretos & Brancos & Pardos & Pretos & Brancos & Pardos & Pretos \\
\hline \multicolumn{10}{|c|}{ Idade (anos) } \\
\hline \multicolumn{10}{|l|}{$<40$} \\
\hline Brancos & 96,6 & 10,0 & 0,0 & 94,6 & 13,2 & 0,0 & 95,6 & 11,5 & 0,0 \\
\hline Pardos & 3,4 & 87,0 & 34,1 & 5,4 & 85,4 & 32,0 & 4,4 & 86,3 & 32,8 \\
\hline Pretos & 0,0 & 2,9 & 65,9 & 0,0 & 1,5 & 68,0 & 0,0 & 2,3 & 67,2 \\
\hline Total (n) & 528 & 239 & 126 & 597 & 205 & 203 & 1.125 & 444 & 329 \\
\hline \multicolumn{10}{|l|}{$40+$} \\
\hline Brancos & 95,1 & 11,6 & 0,0 & 97,4 & 12,3 & 0,3 & 96,5 & 11,9 & 0,2 \\
\hline Pardos & 4,6 & 84,2 & 26,5 & 2,6 & 84,2 & 24,2 & 3,4 & 84,2 & 24,9 \\
\hline Pretos & 0,3 & 4,1 & 73,5 & 0,0 & 3,6 & 75,5 & 0,1 & 3,8 & 74,8 \\
\hline Total (n) & 350 & 241 & 147 & 502 & 253 & 326 & 852 & 494 & 473 \\
\hline \multicolumn{10}{|c|}{ Escolaridade } \\
\hline \multicolumn{10}{|c|}{ 1ㅇ grau } \\
\hline Brancos & 91,9 & 9,6 & 0,0 & 95,5 & 3,3 & 0,6 & 93,5 & 7,0 & 0,4 \\
\hline Pardos & 7,4 & 86,5 & 33,9 & 4,5 & 89,3 & 18,3 & 6,1 & 87,7 & 24,5 \\
\hline Pretos & 0,7 & 3,9 & 66,1 & 0,0 & 7,4 & 81,1 & 0,4 & 5,3 & 75,1 \\
\hline Total (n) & 135 & 178 & 109 & 110 & 122 & 164 & 245 & 300 & 273 \\
\hline \multicolumn{10}{|l|}{ 2o grau } \\
\hline Brancos & 95,5 & 8,8 & 0,0 & 95,3 & 14,1 & 0,0 & 95,4 & 11,3 & 0,0 \\
\hline Pardos & 4,5 & 87,8 & 26,7 & 4,7 & 85,3 & 30,1 & 4,6 & 86,6 & 31,7 \\
\hline Pretos & 0,0 & 3,4 & 73,3 & 0,0 & 0,6 & 69,9 & 0,0 & 2,1 & 68,3 \\
\hline Total (n) & 313 & 205 & 120 & 297 & 177 & 219 & 610 & 382 & 186 \\
\hline \multicolumn{10}{|c|}{ 3o grau ou + } \\
\hline Brancos & 97,7 & 15,8 & 0,0 & 96,2 & 19,0 & 0,0 & 96,8 & 17,7 & 0,0 \\
\hline Pardos & 2,3 & 81,1 & 27,9 & 3,8 & 80,4 & 32,9 & 3,2 & 80,6 & 31,7 \\
\hline Pretos & 0,0 & 3,2 & 72,1 & 0,0 & 0,7 & 67,1 & 0,0 & 1,6 & 68,3 \\
\hline Total (n) & 428 & 95 & 43 & 685 & 153 & 143 & 1.113 & 248 & 186 \\
\hline \multicolumn{10}{|l|}{ Renda* } \\
\hline \multicolumn{10}{|l|}{$<3 \mathrm{SM}$} \\
\hline Brancos & 93,7 & 6,5 & 0,0 & 91,0 & 6,7 & 0,0 & 92,5 & 6,6 & 0,0 \\
\hline Pardos & 5,7 & 88,4 & 25,8 & 9,0 & 88,9 & 24,4 & 7,2 & 88,6 & 24,9 \\
\hline Pretos & 0,6 & 5,0 & 74,2 & 0,0 & 4,4 & 75,6 & 0,3 & 4,8 & 75,1 \\
\hline Total (n) & 159 & 199 & 120 & 133 & 135 & 193 & 292 & 334 & 313 \\
\hline \multicolumn{10}{|l|}{ 3-6 SM } \\
\hline Brancos & 93,9 & 12,4 & 0,0 & 95,5 & 11,7 & 0,0 & 94,8 & 12,0 & 0,0 \\
\hline Pardos & 6,1 & 86,9 & 34,5 & 4,5 & 87,1 & 29,3 & 5,2 & 87,0 & 30,9 \\
\hline Pretos & 0,0 & 0,7 & 65,5 & 0,0 & 1,2 & 70,7 & 0,0 & 0,9 & 69,1 \\
\hline Total (n) & 314 & 153 & 84 & 379 & 163 & 198 & 693 & 316 & 282 \\
\hline \multicolumn{10}{|l|}{$>6 \mathrm{SM}$} \\
\hline Brancos & 98,4 & 12,5 & 0,0 & 97,8 & 20,6 & 0,0 & 98,0 & 17,1 & 0,0 \\
\hline Pardos & 1,6 & 82,3 & 24,0 & 2,2 & 77,0 & 26,4 & 2,0 & 79,3 & 25,6 \\
\hline Pretos & 0,0 & 5,2 & 76,0 & 0,0 & 2,4 & 73,6 & 0,0 & 3,6 & 74,4 \\
\hline Total (n) & 370 & 96 & 50 & 544 & 126 & 110 & 914 & 222 & 160 \\
\hline
\end{tabular}

* Renda familiar per capita em salários mínimos (SM) - valores de 1999. 


\section{Discussão}

Os resultados das questões sobre cor/raça do Estudo Pró-Saúde se inserem no debate sobre o sistema de classificação racial no Brasil, presente na literatura das ciências sociais e ainda incipiente na área da saúde 14,15 . No caso dos estudos sociológicos, a categoria cor/raça tem sido analisada como um elemento estruturante das desigualdades sociais no Brasil ao lado das clivagens de classe, de gênero e de região 20 . Pondera-se que a construção de identidades raciais no país decorre da combinação de diversos aspectos sócio-antropológicos, tais como: grau de instrução, nível de renda, hábitos, aspectos fenotípicos, entre outros. As clássicas discussões de Nogueira 21 sobre classificação étnico/racial no Brasil ilustram esse ponto de vista. Para o autor, diferente do caso norte-americano, é a marca de cor (aparência física) e não a marca de origem (ancestralidade) que fundamenta o sistema de classificação racial no Brasil.

Vale ressaltar que as especificidades das relações raciais na sociedade brasileira são fre qüentemente objeto de análise na definição dos critérios e instrumentos a serem utilizados para o levantamento de dados demográficos no país, com efeitos significativos sobre o perfil sócio-econômico da população. O amplo leque de denominações empregadas no plano da classificação racial, assim como as implicações da utilização por parte do aparato estatal de um sistema de classificação de cor/raça (branco, preto, pardo, amarelo e indígena), adotado pelo IBGE, é alvo de controvérsias entre sociólogos e antropólogos 11,22,23,24. Indaga-se até que ponto as definições utilizadas pelo IBGE retratam as interações sociais que se vinculam a critérios classificatórios bem mais complexos e matizados. A título de ilustração, é importante salientar que desde pelo menos os anos 1950, há uma série de estudos que apontam para a estreita relação entre classificação racial e $s t a$ tus sócio-econômico 21,25,26,27,28,29. Observouse que os segmentos sociais com maior perspectiva de ascensão social teriam uma propensão à identificação com categorias mais próximas à cor/raça branca, configurando assim o processo de "branqueamento". Esse processo tem sido confirmado por algumas pesquisas mais recentes, como as de Silva 11 e de Telles 30. Em contraste, há também indícios crescentes de que escolaridade e renda mais elevadas não implicam, necessariamente, o "embranquecimento" da identificação racial 31,32.

No presente artigo, foram investigados alguns fatores situacionais e relacionais que influenciaram a autoclassificação da cor/raça na população de funcionários públicos fluminenses que participaram da Fase 1 do Estudo PróSaúde. Nesta população, a variabilidade das categorias de autoclassificação identificada nas respostas dos participantes do nosso estudo à pergunta aberta, com a utilização de um amplo repertório de termos, coincide com análises de dados da Pesquisa Nacional por Amostragem de Domicílios (PNAD) de 1976 12,33.

A proporção de participantes que se autoclassificaram como brancos foi semelhante nas respostas às perguntas aberta e fechada. As principais discrepâncias foram observadas entre aqueles que se identificaram como morenos, mestiços e negros ou pretos. Considerando-se a classificação em três categorias raciais (brancos, pardos e pretos), nossos resultados convergem com os achados de outros autores 6,13 no que se refere ao fenômeno do "branqueamento", a despeito das diferenças metodológicas, dado que os estudos citados trabalham com autoclassificação e classificação por terceiros, enquanto que o Estudo Pró-Saúde utilizou dois sistemas de autoclassificação. Foi observado que $28,0 \%$ dos respondentes que se identificaram como negros ou pretos na pergunta aberta, classificaram-se como pardos na pergunta fechada, e $12,0 \%$ dos pardos se classificaram como brancos. Dado que esta tendência tornouse mais evidente entre os estratos de maior escolaridade, pode-se supor que isso se relaciona ao fato de que aqueles situados em posições mais desfavoráveis na escala social têm uma menor expectativa de ascensão social. Como afirma Silva 11 (p. 124), no Brasil existe um duplo movimento: "não só o dinheiro embranquece, como, inversamente, a pobreza escurece".

A variabilidade associada à categoria parda, em particular, representa um dos maiores desafios das classificações sobre a origem étnico/racial no Brasil 34,35. Com base nos dados do Estudo Pró-Saúde, podemos argumentar que a categoria parda, utilizada pelo IBGE, apresenta grande concordância entre as respostas das perguntas aberta e fechada, indicando uma tendência à incorporação e uso da classificação adotada oficialmente. Por outro lado, não se deve perder de vista que a categoria morena é muito comum na autodefinição dos brasileiros, sendo considerada mais consistente que a denominação parda por autores como Marvin Harris 23. A denominação mais adequada para classificar a categoria intermediária entre brancos e pretos, centrada nas categorias parda e morena, tem gerado controvérsias. Segundo Telles 30 e Silva 11, as categorias morena e mestiça apresentam um maior grau de ambigüidade em relação ao termo pardo em função da 
sua abrangência, incluindo desde brancos de cabelos pretos até pardos e mulatos.

Outro aspecto relevante se refere às variações entre o uso das categorias negra e preta. Na classificação aberta, o número de respondentes que se autoclassificou como negro (227 homens e 474 mulheres) é muito superior ao daqueles que se definiram como pretos (46 homens e 55 mulheres). Pode-se especular que aqueles que se classificaram como negros revelam uma maior aproximação com uma identidade racial de origem cultural e/ou política contemporânea.

É interessante notar que a variabilidade da classificação racial não parece ser um problema brasileiro apenas. Recentemente, Kaplan \& Bennett 18 comentaram que o primeiro grande desafio para a incorporação de cor/raça às publicações biomédicas (mesmo a norte-americana) é admitir que a identidade racial não é fixa nem imutável, e ainda que sua validade e confiabilidade são limitadas. Sugere-se que a escolha e o uso combinado de métodos classificatórios (autoclassificação e classificação por terceiros), deve depender do objeto de estudo (Williams, 2002, apud Kaplan \& Bennett 18), embora a autoclassificação venha sendo considerada como "padrão-ouro" por alguns autores 16,17.

Uma vez que consideramos raça/etnia como uma construção sociocultural, o conceito de "padrão-ouro" não parece ser adequado. No caso do Brasil, tanto a classificação realizada por entrevistador como a autoclassificação podem "branquear" ou "escurecer" a categoria do participante, dependendo de contextos mais amplos (como a região do país) e da situação individual (posição social). Por exemplo, em inquérito nacional, a classificação realizada por entrevistadores mostrou-se mais "branqueadora” da população mais rica do que a autoclassificação 30 .

A escolha da estratégia e do sistema de classificação deve levar em consideração vanta- gens e desvantagens de cada uma das alternativas, além dos objetivos da investigação. Por exemplo, ao utilizar as categorias do IBGE, ganha-se em termos de comparabilidade com outros estudos, mas é preciso ponderar a possibilidade de tendência ao "branqueamento", especialmente nos estratos de melhor posição sócio-econômica. Vale ressaltar, como apontam Kaplan \& Bennett 18, que qualquer conjunto de opções de resposta é apenas uma aproximação do que é socialmente considerado como categorias étnico/raciais. Assim, os pesquisadores devem ser cautelosos ao inferir que os indivíduos pertencem a estas categorias de maneira definitiva, ou que cada uma dessas categorias agrega um grupo absolutamente homogêneo.

As análises dos dois sistemas de autoclassificação (perguntas aberta e fechada) do Estudo Pró-Saúde indicam resultados distintos que, potencialmente, podem influenciar as análises epidemiológicas do estudo. Tendo em vista os argumentos assinalados acerca da variabilidade e fluidez do sistema de classificação, sugere-se que a pergunta aberta, quando viável, é mais adequada do que a autoclassificação fechada, já que permite uma visão mais acurada das variações na autopercepção dos indivíduos, podendo ainda captar as mudanças acerca dos usos das categorias raça e cor e dos processos de construção de identidades étnico/raciais.

A utilização do recorte étnico/racial nos estudos de saúde nos parece essencial já que oferece a oportunidade de reunir evidências epidemiológicas, ainda escassas, a respeito de grupos historicamente desfavorecidos do ponto de vista sócio-econômico. A produção de informação sobre a saúde desses diversos grupos pode permitir a configuração de um quadro mais detalhado a respeito das iniqüidades sociais e das desigualdades em saúde no Brasil, e a formulação de políticas públicas informadas pelas especificidades da sociedade brasileira. 


\section{Resumo}

Variações nos sistemas de classificação da cor/raça e a dependência contextual de sua aplicação são alguns dos desafios para a realização de estudos de saúde com recorte étnico/racial no Brasil. As respostas a duas abordagens distintas para autoclassificação de raçaquestão fechada (IBGE) e questão aberta - foram comparadas em um estudo de coorte dos funcionários de uma universidade no Rio de Janeiro. De acordo com a pergunta fechada, 54,0\% dos 3.717 participantes classificaram-se como brancos, 30,0\% como pardos e 16,0\% como pretos. Segundo a pergunta aberta, essas proporções foram 53,0\%, 25,0\% e 22,0\%, respectivamente, agrupando-se os termos moreno, mestiço e mulato na categoria parda. Apesar da concordância elevada (kappa = 0,80; IC95\%: 0,78-0,82), proporções não despreziveis de participantes negros ou pardos (pergunta aberta) escolheram categorias mais "claras" entre as opções do IBGE. A utilização do recorte étnico/racial nos estudos de saúde pode não apenas revelar dados sobre as desigualdades sociais no Brasil, mas também contribuir para a formulação de políticas na área da saúde pública informadas pelas especificidades da sociedade brasileira.

Grupos Étnicos; Estudos de Coortes; Iniqüidade na Saúde

\section{Colaboradores}

M. C. Maio e S. Monteiro foram responsáveis pela fundamentação teórica (sócio-antropológica) e participaram da categorização e análise dos dados e da discussão dos resultados. D. Chor contribuiu no planejamento e condução do estudo, na análise de dados, na descrição do método e resultados, e na discussão dos resultados. E. Faerstein contribuiu no planejamento e condução do estudo, na análise de dados e na discussão dos resultados. C. S. Lopes participou do planejamento e condução do estudo e da discussão dos resultados.

\section{Agradecimentos}

Agradecemos às sociólogas Celi Scalon e Márcia Lima pelas críticas e sugestões.

\section{Referências}

1. Monteiro S. Desigualdades em saúde, raça e etnicidade. In: Monteiro S, Sansone L, organizadores. Etnicidade na América Latina: um debate sobre raça, saúde e direitos reprodutivos. Rio de Janeiro: Editora Fiocruz; 2004. p. 45-56.

2. Coimbra Jr. C, Santos RV. Saúde, minorias e desigualdade: algumas teias de inter-relações com ênfase nos povos indígenas no Brasil. Ciênc Saúde Coletiva 2000; 5:125-32.

3. Candwell K. Fronteiras da diferença: raça e mulher no Brasil. Estudos Feministas 2000; 8:91-108.

4. Monteiro S, Maio MC. Saúde da população negra: questões e desafios. In: Anais do VII Congresso Latino-Americano de Ciências Sociais e Saúde; no prelo.

5. Programa das Nações Unidas para o Desenvolvimento/Organização Pan-Americana da Saúde. Política nacional de saúde da população negra: uma questão de eqüidade. Brasília: Programa das Nações Unidas para o Desenvolvimento/Organização Pan-Americana da Saúde; 2001.

6. Hasenbalg CA. Discriminação e desigualdades raciais no Brasil. Rio de Janeiro: Edições Graal 1979.

7. Hasenbalg CA, Silva NV. Estrutura social, mobilidade e raça. São Paulo: Vértice; 1988.

8. Hasenbalg CA, Silva NV, Lima M. Cor e estratificação social. Rio de Janeiro: Editora Contracapa 1999.

9. Krieger N. Discrimination and health. In: Berkman L, Kawachi I, editors. Social epidemiology. Oxford: Oxford University Press; 2000. p. 36-68.

10. Williams D. The monitoring of racia/ethnic status in the USA: data quality issues. Ethn Health 1999; 4:121-37.

11. Silva NV. Uma nota sobre "raça social" no Brasil. In: Hasenbalg CA, Silva NV, Lima M, organizadores. Cor e estratificação social. Rio de Janeiro: Editora Contracapa; 1999. p. 107-25.

12. Silva NV. Cor e processo de realização socioeconômica. In: Hasenbalg CA, Silva NV, organizadores. Estrutura social, mobilidade e raça. São Paulo: Vértice; 1988. p. 144-63.

13. Telles E. Racismo à brasileira: uma nova perspectiva sociológica. Rio de Janeiro: Relume-Dumará 2003.

14. Fuchs SC, Guimarães SM, Sortica C, Wainberg F, Dias KO, Ughini M, et al. Reliability of race assessment based on the race of the ascendants: a cross-sectional study. BMC Public Health 2002; 2:1-5.

15. Fuchs SC, Victora CG, Wannmacher L, Gonçalves C, Barbosa A, Menta L, et al. Reliability of environmental, biological and health characteristics of infants from developing country. Int J Epidemiol 1996; 25:1005-8.

16. Boehmer U, Kressin NR, Berlowitz DR, Chris tiansen CL, Kazis LE, Jones JA. Self-reported vs. administrative race/ethnicity data and study results. Am J Public Health 2002; 92:1471-3.

17. Kaufman JS. How inconsistencies in racial classification demystify the race construct in public health statistics. Epidemiology 1999; 10:101-13.

18. Kaplan JB, Bennett $T$. Use of race and ethnicitiy in 
biomedical publication. JAMA 2003; 289:2709-16.

19. Streiner DL, Norman GR. Health measurement scales: a practical guide to their development and use. 28th Ed. Oxford: Oxford University Press; 1995.

20. Costa S. A construção sociológica da raça no Brasil. Estud Afro-Asiát 2002; 24:35-61.

21. Nogueira O. Preconceito racial de marca e preconceito racial de origem (sugestão de um quadro de referência para a interpretação do material sobre relações raciais no Brasil). In: Anais do XXXI Congresso Internacional dos Americanistas. São Paulo: Editora Anhembi; 1955. p. 409-34.

22. Schwartzman S. Fora de foco: diversidade e identidades étnicas no Brasil. Novos Estudos CEBRAP 1999; 55:83-96.

23. Harris M, Consorte JG, Lang J, Byrne B. Who are the whites? Imposed census categories and the racial demography of Brazil. Soc Forces 1993; 72: 451-62.

24. Telles E. Who are the morenas? Soc Forces 1995; 73:1609-11.

25. Wagley C. On the concept of social face in the Americas. In: Heath DB, Adams RN, editors. Contemporary cultures and societies of Latin America. New York: Random House; 1965. p. 531-45.

26. Wagley C, editor. Race and class in rural Brazil. Paris: United Nations Educational, Scientific and Cultural Organization; 1952.

27. Harris M. Racial identity in Brazil. Luso-Brazilian Review 1964; 1:21-8.
28. Harris M. Referencial ambiguity in the calculus of Brazilian racial identity. Southern Journal of Anthropology 1970; 26:1-14.

29. Harris M, Kotak C. The structural significance of Brazilian categories. Sociologia (São Paulo) 1963; 25:203-8.

30. Telles E. Racial ambiguity among the Brazilian population. Ethn Racial Stud 2002; 25:415-41.

31. Figueiredo A. Novas elites de cor: estudos sobre profissionais negros em Salvador. São Paulo: Annablume Editora/Centro de Estudos Afro-Asiáticos, Universidade Cândido Mendes; 2002.

32. Teixeira MP. Negros na universidade: identidade e trajetórias de ascensão social no Rio de Janeiro. Rio de Janeiro: Editora Pallas; 2003.

33. Maggie Y. Aqueles a quem foi negada a cor do dia: as categorias de cor e raça na cultura brasileira. In: Maio MC, Santos RV, organizadores. Raça, ciência e sociedade. Rio de Janeiro: Editora Fiocruz/ Centro Cultural Banco do Brasil; 1996. p. 225-34.

35. Schwarcz LKM. Questão racial e etnicidade In Miceli S, organizador. O que ler na ciência social brasileira (1970-1995). São Paulo. Editora Sumaré/Associação Nacional de Pós-graduação em Ciências Sociais/Coordenação de Aperfeiçoamento de Pessoal de Nível Superior; 1999. p. 267-325.

36. Silva NV. Morenidade: modos de usar. Estud AfroAsiát 1996; 30:79-96.

Recebido em 28/Jan/2004

Versão final reapresentada em 08/Jul/2004

Aprovado em 04/Ago/2004 